\title{
MICROMAMÍFEROS, TAFONOMÍA Y AMBIENTES DURANTE LOS ÚLTIMOS 3.800 AÑOS A.P. EN EL VALLE MEDIO DEL RÍO GRANDE, MENDOZA, ARGENTINA
}

\author{
MICROMAMMALS, TAPHONOMY AND ENVIRONMENTS SINCE 3,800 YR. BP AT \\ THE MIDDLE VALLEY OF GRANDE RIVER, MENDOZA, ARGENTINA
}

\author{
Fernando J. Fernández ${ }^{1,2}$, Sergio M. Rudzik ${ }^{1}$, Gustavo A. Neme $e^{3,4}$ y Luciano J.M. De Santis ${ }^{1}$
}

\begin{abstract}
Desde una perspectiva tafonómica y paleoambiental se estudiaron restos de micromamíferos recuperados de dos sitios arqueológicos que abarcan un período temporal desde 3.800 años a.p. hasta el siglo XX y se ubican en el curso medio del río Grande, sudoeste de la provincia de Mendoza (Cueva de Luna y Alero Puesto Carrasco). La presencia de marcas de digestión ligera y moderada indica que los micromamíferos fueron depositados por aves strigiformes, posiblemente Tyto alba. Esto contrasta con el modelo de intensificación de los recursos planteado para los grupos cazadores-recolectores que habitaron el sur de Mendoza hacia el 2.000 a.p. Los conjuntos zooarqueológicos mostraron cierta estabilidad taxonómica, presentando especies patagónicas (p.ej. Lestodelphys halli, Reithrodon auritus y Euneomys chinchilloides) y otras afines al desierto del Monte (p.ej. Galea leucoblephara, Akodon iniscatus y Calomys musculinus), en contraste con las muestras actuales donde principalmente se hallaron especies patagónicas, señalando un paleoambiente más árido y cálido que el actual.
\end{abstract}

Palabras claves: micromamíferos, tafonomía, paleoambientes, zooarqueología del río Grande, Mendoza.

This paper describes micromammal samples from two archaeological sites spanning a period from 3,800 yr. BP until the 20th century, and located in the middle basin of the river Grande, in the south-west of the Mendoza Province (Cueva de Luna and Alero Puesto Carrasco). Also, the taphonomic and paleoenvironmental conditions are assessed. The recording of light and moderate digestive traces indicates that the micromammals of both samples were deposited by strigiform birds, possibly Tyto alba. This contrasts with the model of intensification of resources proposed for the hunter-gatherers who inhabited southern Mendoza toward 2,000 yr. BP. The archaeofaunistic assemblages showed some taxonomic stability, including Patagonian species (e.g., Lestodelphys halli, Reithrodon auritus and Euneomys chinchilloides) and others associated with the Monte desert (e.g., Galea leucoblephara, Akodon iniscatus and Calomys musculinus), contrary to recent samples, which mainly found Patagonian species, suggesting a more arid and warm palaeonvironment than recent times.

Key words: Small mammals, taphonomy, paleoenvironments, zooarchaeology of the river Grande, Mendoza.

El área que comprende la cuenca media del río Grande se encuentra en el sudoeste de la provincia de Mendoza (Dpto. de Malargüe). Incluye sectores pedemontanos ubicados entre la cordillera frontal al oeste y la región volcánica de La Payunia al este (Capitanelli 1972). Fitogeográficamente se enmarca en la provincia patagónica (sensu Cabrera 1976). Esta unidad se caracteriza por la presencia de estepas arbustivas y herbáceas, estas se desarrollan sobre suelos basálticos, arenosos, pedregosos y pobres en materia orgánica y en sectores de mayor humedad llamados regionalmente "vegas" (Cabrera 1976;
Capitanelli 1972). En los valles medio e inferior del río Grande se observan disparidades térmicas bien marcadas, con veranos templados e inviernos muy fríos, donde se registran intensos vientos provenientes del anticiclón del Pacífico, descargando precipitaciones níveas en el orden de los $600 \mathrm{~mm}$ (Capitanelli 1972). Hacia el extremo oriental de la provincia tiene lugar el desierto del Monte, presentando un clima más cálido y árido, con un promedio anual de precipitaciones estivales de 200 mm, influenciado por el anticiclón del Atlántico. La vegetación predominante de esta unidad es de

Facultad de Ciencias Naturales y Museo, UNLP, Calle 64 s/n, La Plata, 1900, Argentina. fernandezf77@yahoo.com.ar; martinrudzik@hotmail.com; desantis@fcnym.unlp.edu.ar

2 CONICET Consejo Nacional de Investigaciones Científicas y Técnicas, Av. Rivadavia 1917, CABA, 1033, Argentina.

3 CONICET-IANIGLA Instituto Argentino de Nivología, Glaciología y Ciencias Ambientales, Av. Ruiz Leal s/n Parque General San Martín, Mendoza, 5500, Argentina.

4 Museo de Historia Natural de San Rafael. Av. Ballofet s/n, Parque Mariano Moreno. San Rafael, 5600, Argentina. gneme@mendoza-conicet.gob.ar 
tipo estepa arbustiva xerófila, sammófila o halófila (Cabrera 1976). Esta compleja situación en la dinámica ambiental de la cuenca media del río Grande, debido a que ciertos elementos arbustivos de esta unidad (p.ej., Larrea spp.) ingresan hacia el oeste por medio del cauce del río, aunque sin extenderse demasiado de sus márgenes (Martínez Carretero 2006; Roig et al. 2000).

Este esquema fitogeográfico del sur de Mendoza presenta en un gradiente altitudinal de oeste-este el pasaje de condiciones patagónicas al desierto del Monte. Estas transiciones ambientales ocurren en distancias relativamente cortas (Figura 1) y también afectan a la composición de la fauna asociada (Roig 1972). Los micromamíferos constituyen una clara manifestación de esta situación (Fernández 2012a; Pardiñas et al. 2008). Esto se debe a que numerosas especies de micromamíferos son sensibles a los cambios en la composición y abundancia de la vegetación (p.ej., Pardiñas 1999a; Teta et al. 2014). En tal sentido, las características estenoicas de numerosas especies de micromamíferos, su alta sensibilidad a los cambios ambientales y su abundancia en el registro arqueológico, principalmente del tipo cueva o refugio rocoso, permiten su utilización como indicadores de las características ambientales en el pasado (p.ej., Andrews 1990, 1995; Fernández 2012a; Fernández et al. 2009; Pardiñas 1999a; Teta et al. 2014).

El cauce medio del río Grande ha sido explorado arqueológicamente desde las décadas de 1970 y 1980, donde surgieron los trabajos pioneros del Prof. Mariano Gambier, en el sitio Gruta el Manzano (Gambier 1980, 1985). A mediados de 1980 y durante la década de 1990 se ampliaron los muestreos arqueológicos, dando origen a numerosas investigaciones dirigidas principalmente por el Dr. Víctor Durán, en los sitios Cañada de Cachi, Cueva de Los Indios, Cueva de Luna y Alero Puesto Carrasco (p.ej. Durán 1997, 2004; Durán y Ferrari 1991; Durán et al. 1999; Neme et al. 1995, 1999). Recientemente se profundizaron los estudios en el área (Neme et al. 2011) incluyendo investigaciones geoarqueológicas (Durán y Mikkan 2009), arqueobotánicas (Llano 2011) y zooarqueológicas (Fernández 2012a; Giardina 2010a, 2010b; Neme et al. 2011). Sin embargo, hasta el momento solo se cuenta con datos paleoambientales específicos provenientes de la cuenca superior del río Grande (Espizua 2003, 2005), de modo que la información emanada del registro de micromamíferos de los sitios ubicados en la cuenca media cobrarán mayor relevancia. En relación con esto último, Fernández (2012a), en el marco de su tesis doctoral, realizó un estudio tafonómico y paleoambiental de micromamíferos recuperados del sitio Cueva de Luna.

Para el sur de Mendoza se ha planteado un cambio en el uso de la fauna hacia mediados del Holoceno tardío (últimos 2.000 años a.p.), el que muestra un uso creciente de taxones de tamaños más pequeños y por tanto de menor rendimiento económico (Neme 2007). El cuerpo de conocimiento donde descansa esta asunción es la teoría de la depredación óptima, sobre la base de la ecología evolucionista (p.ej., Bettinger 1991; Broughton 1994; Neme 2007). En este contexto surge el concepto de intensificación de los recursos como una forma para explicar los cambios en la subsistencia de las poblaciones humanas del sur de Mendoza en el tiempo (p.ej., Neme 2007; Neme et al. 2011). Según plantearon estos autores, este proceso tuvo lugar por una reducción de los rangos de movilidad, variaciones tecnológicas y un desbalance entre la capacidad sustentadora del ambiente y el aumento demográfico de los grupos cazadores-recolectores. De modo que la sobreexplotación del ambiente pudo reducir la disponibilidad de los recursos altamente ranqueados como el guanaco (Lama guanicoe) y las aves corredoras de gran tamaño (Rhea americana y $R$. pennata) y aumentar el aprovechamiento de especies de menor retorno calórico, como algunas plantas y vertebrados de menor tamaño (p.ej., Fernández et al. 2009; Giardina 2010a, 2010b; Llano 2011; Neme 2007; Neme et al. 2011).

Dentro de este modelo regional se desprende la posibilidad de que la amplitud de dieta de los grupos humanos haya aumentado hasta incorporar a los micromamíferos. En este sentido, el estudio tafonómico de los restos de micromamíferos provenientes de sitios arqueológicos cobra singular importancia, no solo desde la formación de los conjuntos, sino también en el papel que los micromamíferos puedan haber jugado en la economía de los cazadores-recolectores (Fernández 2012a; Fernández y De Santis 2013). Una buena comprensión de las historias tafonómicas de los conjuntos de micromamíferos permitirán discutir con mayor seguridad el origen de las muestras, su probable utilización como recurso alimenticio por parte de los grupos humanos prehispánicos y la integridad de los depósitos arqueológicos en general (Fernández 2012a). 


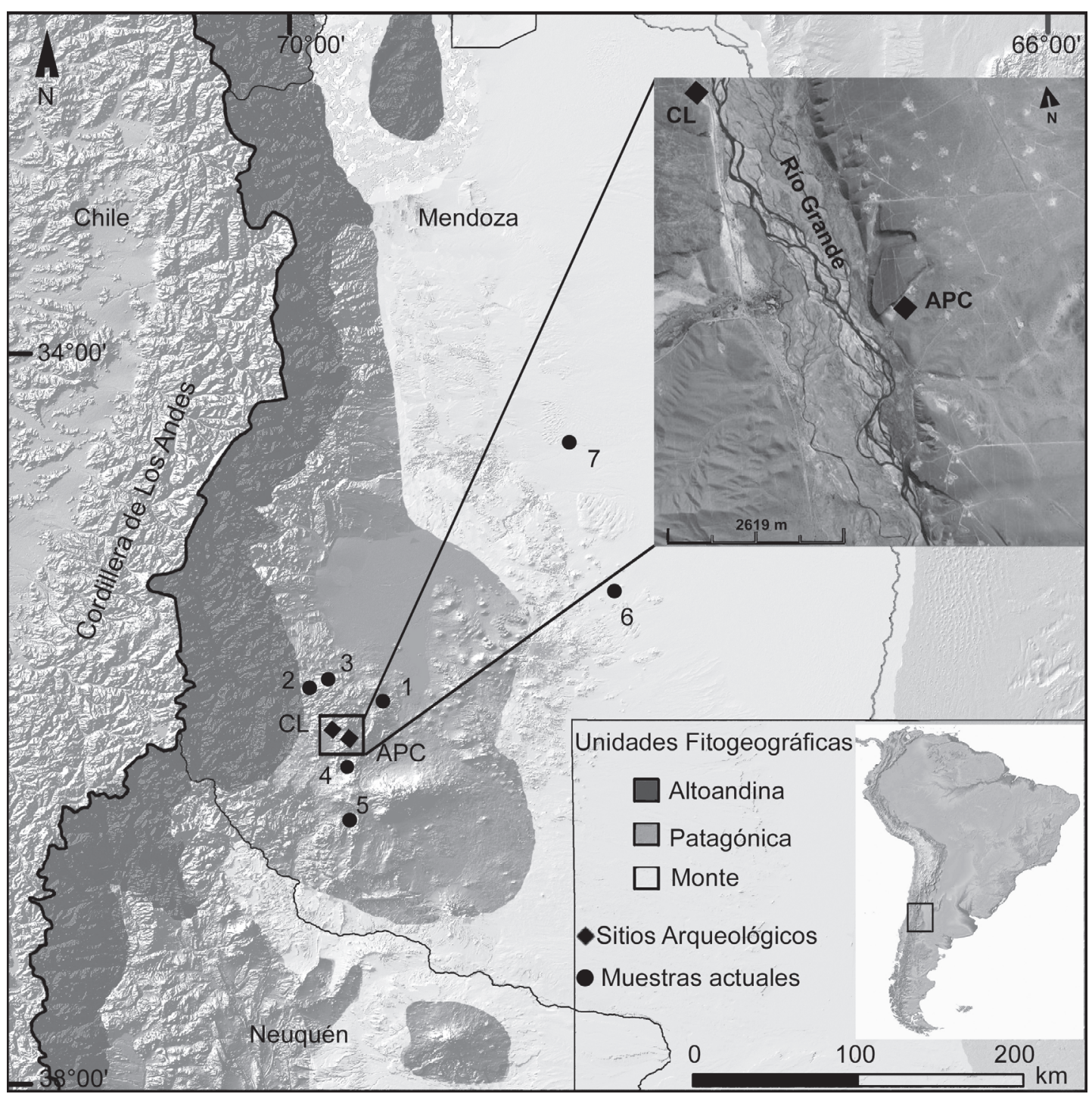

Figura 1. Mapa de Mendoza con las unidades fitogeográficas (sensu Cabrera 1976) y la ubicación de los sitios arqueológicos y las muestras actuales. Los números de las localidades se definen en la Tabla 1.

Map of Mendoza with phytogeographic units (sensu Cabrera 1976), showing the location of the archaeological sites and recent samples. The numbers of the localities are defined in Table 1.

En este trabajo se analizará la historia tafonómica de los conjuntos de micromamíferos recuperados de dos sitios arqueológicos (Cueva de Luna y Alero Puesto Carrasco) ubicados en el curso medio del río Grande, sudoeste de la provincia de Mendoza. Se hará énfasis en la determinación de series de atributos que permitan diferenciar los agentes causantes de la acumulación y los procesos postdepositacionales sufridos por los restos. Este análisis será útil para discutir el rol que cumplieron los micromamíferos en la subsistencia de los grupos humanos que habitaron los sitios. En tal sentido, se pondrá a prueba la hipótesis de intensificación de los recursos planteada para el sur de Mendoza hacia el 2.000 a.p.

Se evaluará la distribución espacio-temporal de los taxones de micromamíferos recuperados de ambos sitios arqueológicos como una vía de acercamiento hacia una mayor comprensión de los paleoambientes del cauce medio del río Grande durante los últimos 3.800 años. Se esperan cambios en la frecuencia y diversidad de los taxones de micromamíferos en relación con las variaciones en la estructura ambiental del área. 


\section{Sitios Arqueológicos}

\section{Cueva de Luna (CL)}

Está ubicado en la margen derecha del río Grande, a $1.500 \mathrm{~m}$ de su confluencia con el arroyo El Manzano (3604’33,7' S, 6943’22,9” O, 1.300 msm, Figura 1) (Durán 1997, 2004; Durán y Ferrari 1991; Neme et al. 1995). La cueva, constituida por la erosión de una formación basáltica, presenta 9 $\mathrm{m}$ de frente y $8,5 \mathrm{~m}$ de profundidad. Se excavó una trinchera con orientación norte a sur, totalizando una superficie de 3,5 m² (Durán 1997, 2004; Durán y Ferrari 1991; Neme et al. 1995). Presenta un paquete sedimentario de ca. $60 \mathrm{~cm}$ de potencia, donde se distinguieron tres capas naturales (Durán 1997, 2004; Durán y Ferrari 1991). En la Tabla 1 se detallan las principales características estratigráficas, cronológicas y culturales del sitio.

\section{Alero Puesto Carrasco (APC)}

Es un abrigo rocoso localizado a $4 \mathrm{~km}$ al sudeste de CL (36 06'13"'S, 6941'32”' O, 1.300 msm, Figura 1) (Durán y Ferrari 1991). Se cuenta con $5 \mathrm{~m}^{2}$ de área excavada. Presenta una estratigrafía compuesta de sedimentos relativamente homogéneos, limosos a limo-arenosos, con porcentajes altos de lapilli y cenizas volcánicas (Durán 1997; 2004; Durán et al. 1999). En la Tabla 1 se detallan las principales características estratigráficas, cronológicas y culturales del sitio.

\section{Materiales y Métodos}

Los micromamíferos $(<1 \mathrm{~kg})$ se recuperaron en zaranda de malla de $2 \mathrm{~mm}$ y las determinaciones taxonómicas se realizaron utilizando ejemplares de comparación de la Colección de Mamíferos del Museo de La Plata y fuentes bibliográficas (p.ej., Fernández et al. 2011a; Ballejo et al. 2011; Pearson 1995). Los materiales fueron depositados en la colección de Arqueología del Museo de Historia Natural de San Rafael (MHNSR), bajo los siguientes números: CL (MHNSR-000009) y APC (MHNSR-000010).

\section{Muestras arqueológicas}

Se calculó el número de especímenes identificados por taxón (NISP) y número mínimo de individuos (MNI) (cf. Grayson 1984). Los restos de micromamíferos de ambos sitios (CL: NISP=230,
$\mathrm{MNI}=127$; $\mathrm{APC}: \mathrm{NISP}=57, \mathrm{MNI}=23$ ) se evaluaron tafonómicamente siguiendo la metodología propuesta por Andrews (1990) y Fernández-Jalvo y Andrews (1992). En este marco se observaron las marcas de digestión gástrica y grado de fractura. También se evaluaron variables antrópicas como marcas de corte, patrones de termoalteración (cf. Medina et al. 2012) y dos índices tafonómicos: (1) Índice de Tamaño (IT) $=($ MNI chicos $[$ mamíferos con una longitud corporal $<120 \mathrm{~mm}]+$ MNI medianos [120-170 mm] / MNI grandes [170-250 mm] + MNI muy grandes [ $>250 \mathrm{~mm}]$ ) x 100 . Valores por encima de 100 indican acción de aves rapaces nocturnas (Strigiformes) y debajo de este número podría indicar actividad humana. (2) Índice de Predictabilidad $(\mathrm{IP})=(\mathrm{MNI}$ gregarios $+\mathrm{MNI}$ coloniales + MNI agrupados) / (MNI crípticos + MNI solitarios) x 100 . Valores arriba de 100 podrían sugerir acumulaciones producidas por humanos y aves rapaces diurnas (Accipritiformes, Falconiformes) y por debajo de este número podrían indicar acción de rapaces nocturnas. Los roedores histricognatos se incluyen en las categorías de grandes, muy grandes, coloniales, gregarios y agrupados; y los roedores sigmodontinos y marsupiales corresponden a las categorías de medianos, chicos, crípticos y solitarios (Pardiñas 1999a, 1999b).

Además se evaluaron variables postdepositacionales como meteorización, pisoteo, marcas de raíces, impresiones de óxido de manganeso, abrasión por transporte hídrico y corrosión sedimentaria (cf. Andrews 1990; Fernández-Jalvo y Andrews 2003; Korth 1979). Sin embargo, numerosas variables tafonómicas propuestas por los autores mencionados no pudieron analizarse (p.ej., abundancia relativa de partes esqueletales), debido a que no se recuperaron los elementos postcraneales y los dientes aislados. Estos sesgos metodológicos se deben a que estos sitios fueron excavados en épocas donde los interrogantes acerca de los micromamíferos provenientes de excavaciones arqueológicas se orientaban únicamente a registrar qué taxones se encontraban en los sitios arqueológicos y por tal razón solo se exhumaban restos craneanos y mandibulares, los que son los más indicados para dicha tarea.

Para este estudio los Componentes I, II y III de CL, debido a su homogeneidad y por corresponder a un período histórico, se tomaron como un único conjunto y los Componente IV y V se analizaron por separado. Es admisible notar que una parte del conjunto recuperado del sitio en el mismo contexto 


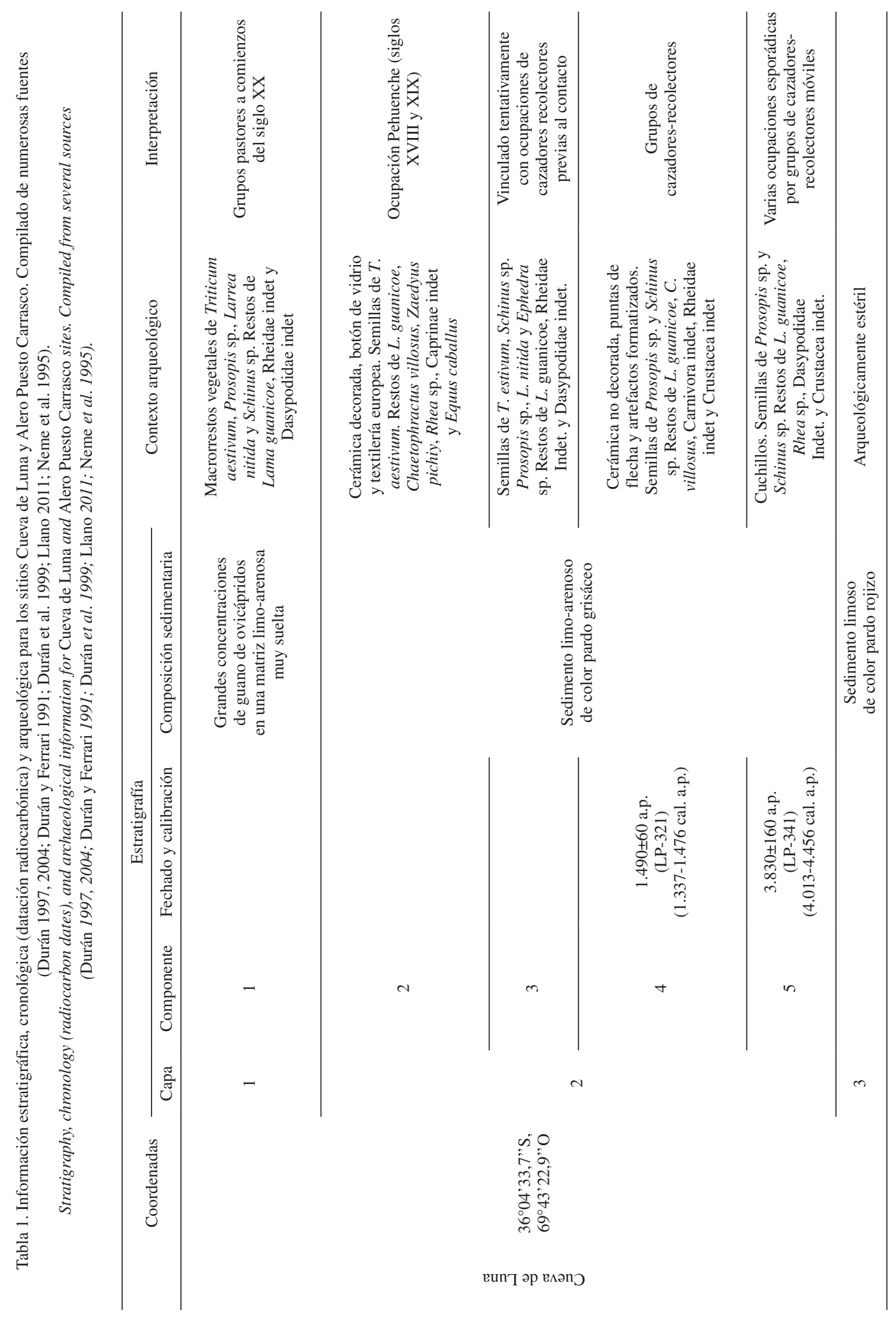




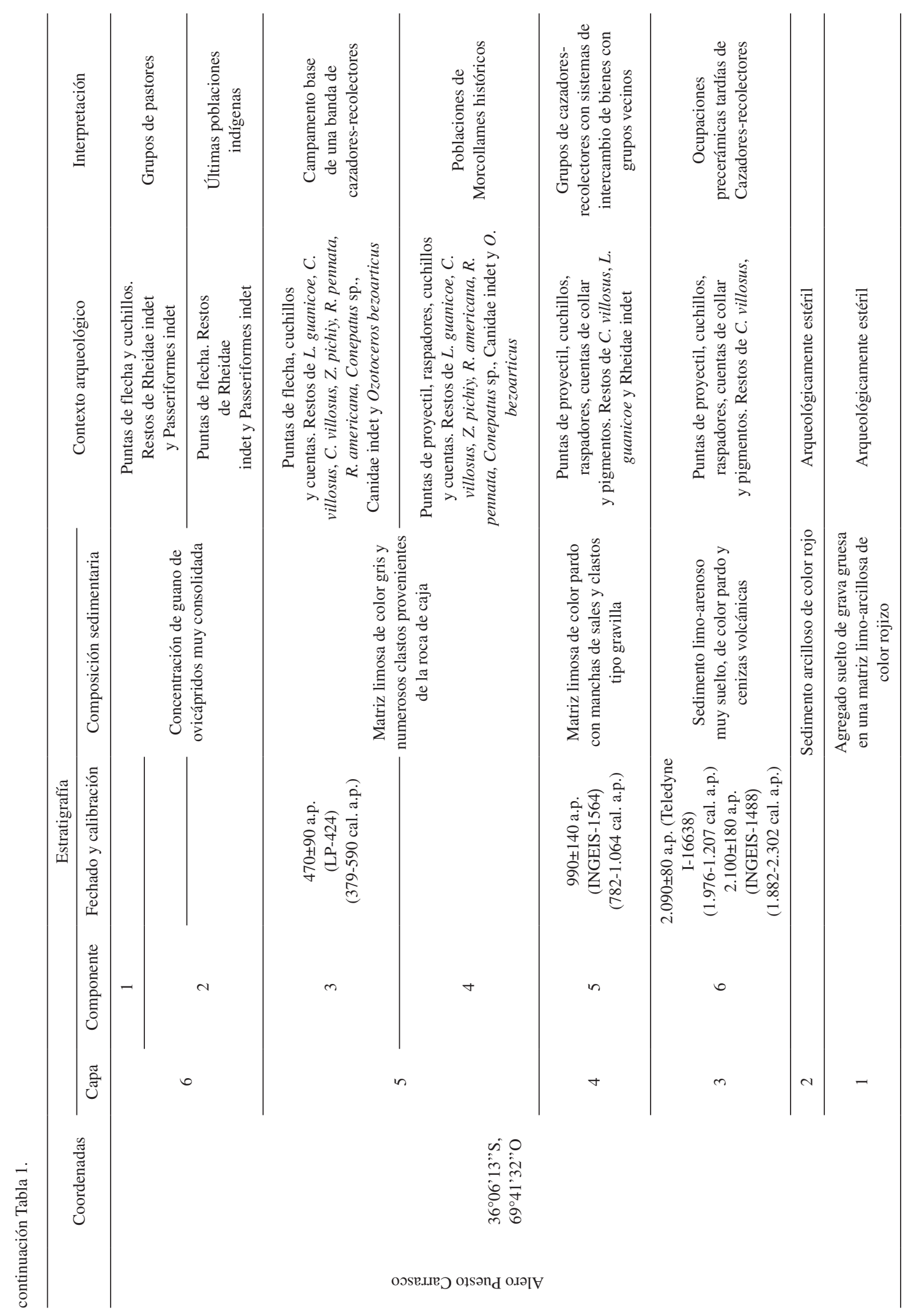


de excavación no se pudo analizar tafonómicamente debido a que por el momento esa muestra se encuentra extraviada. La misma había sido determinada taxonómicamente por Oliver Pearson y la lista de micromamíferos, sin interpretaciones tafonómicas ni paleoambientales, fue publicada por Neme et al. (1995) basada en el cálculo de NISP. Por ende, el cómputo de MNI de esta muestra se realizó en forma estimada, dividiendo por dos a los valores de NISP, considerando que las determinaciones se hicieron a base de maxilares y mandíbulas. Para el caso de APC, debido a la escasez de materiales, se tomaron todos los conjuntos en una sola unidad de análisis.

\section{Muestras actuales}

Los conjuntos de micromamíferos de los sitios arqueológicos se compararon con las comunidades de micromamíferos vivientes en el área, mediante la utilización de muestras de egagrópilas de aves rapaces provenientes de diferentes localidades del sur de Mendoza (Figura 1): (1) Volcán Malacara (Tyto alba, 35 47'26"S y 69²9'13"O, $1.700 \mathrm{msm}$, MHNSR-0101, Fernández 2012a); (2) $10 \mathrm{~km}$ al Oeste de Bardas Blancas (ave rapaz indet., 35 $52^{\prime} 28^{\prime \prime} \mathrm{S}$, 6952’46” O, 1.152 m, Centro Nacional Patagónico CNP-E-289, Pardiñas et al. 2008); (3) Caverna de las Brujas (ave rapaz indet., 3545’ S, 6949’ O, 1.800 msm, Laboratorio de Geoarqueología de la Facultad de Filosofía y Letras UNCUYO, Gasco et al. 2006); (4) La Pasarela (ave rapaz indet., $36^{\circ} 18^{\prime} 46^{\prime \prime} \mathrm{S}$, 6940'03" O, 1.250 msm, CNP-E-198, Pardiñas et al. 2008); (5) Los Frisos (ave rapaz indet., $36^{\circ} 28^{\prime} 20^{\prime \prime}$ S, 69³8'54" O, 1.101 msm, CNP-E-199, Pardiñas et al. 2008); (6) Agua de La Mula (Geranoaetus melanoleucus, $35^{\circ} 22^{\prime} 34,6^{\prime \prime} \mathrm{S}, 68^{\circ} 14^{\prime} 42,1^{\prime \prime} \mathrm{O}, 970$ msm, MHNSR-0093, Fernández 2010, 2012a); (7) Gruta del Indio (Bubo virginianus, 344ㄴ ${ }^{\circ}$ S, $68^{\circ} 21^{\prime}$ O, 700 msm, MHNSR-0095, Fernández 2012a).

Estas aves rapaces se alimentan principalmente de micromamíferos y producen conjuntos óseos y dentarios con modificaciones tafonómicas con un grado de destrucción de leve a moderada (Andrews 1990; Gómez 2005; Fernández 2012a). Sin embargo, algunos sesgos podrían afectar a estas muestras, debido a las diferencias entre las dimensiones de sus territorios de caza y los hábitos (diurnos / nocturnos; solitarios / coloniales) y tamaños (chicos / medianos / grandes) de las presas que consumen. A pesar de los sesgos mencionados, como se asume que sus comportamientos tróficos no han variado en el tiempo, la base de datos de los micromamíferos recolectados en el área de estudio es una sólida estructura para comparar con aquellos recuperados de los sitios arqueológicos (p.ej., Fernández 2012a; Teta et al. 2014). De este modo, estas aves rapaces podrían considerarse buenos estimadores de la comunidades de micromamíferos de un área determinada.

Finalmente se realizaron cálculos de riqueza y diversidad taxonómica y análisis de componentes principales, para explorar las relaciones entre las muestras arqueológicas y las muestras actuales de micromamíferos. Dichos cómputos se efectuaron a base del MNI, usando el programa estadístico PAST (PAleontological STatistics), versión 2.07 (Hammer 1999-2011).

\section{Resultados y Discusión}

\section{Tafonomía}

En ambos sitios se encontraron restos con evidencias de corrosión digestiva, mayormente comprendidos en la categoría de modificación ligera (Figura 2a) y en menor medida en la categoría moderada (Figura 2b) (APC: Ligera $=10,5 \%$, Moderada $=3,5 \%$; CL Componente I, II y III: Ligera $=8,9 \%$, Componente IV: Ligera $=11,8 \%$, Componente V: Ligera $=16,7 \%$ ). Asimismo, se registraron escasos elementos con restos de pelos de egagrópilas $(\mathrm{APC}=2 ; \mathrm{CL}$ Componente $\mathrm{V}=1$; Figura 2c). Casi todos los restos estaban fracturados (APC $=96,5 \% ; C L=99,6 \%$ ).

Se destaca que no se registraron restos con marcas de corte y solo dos elementos del Componente I, II y III y del Componente V de CL estaban termoalterados (Figura 2d), sin el patrón característico de acción humana (i.e., quemado del extremo de las mandíbulas, premaxilares e incisivos; cf. Medina et al. 2012). Los índices de tamaño (IT) y predictibilidad (IP) acusaron valores que indican dominancias de roedores chicos, crípticos y solitarios, consistente con la acción depredadora de aves rapaces nocturnas, a excepción del Componente I, III y III de CL, el que expresó valores contrarios por la abundancia de roedores histricognatos (APC: $\mathrm{IT}=360, \mathrm{IP}=27,8$; CL Componente I, II y III: IT $=43,2, \mathrm{IP}=231,6$, Componente IV: IT $=150, \mathrm{IP}=66,7$, Componente V: $\mathrm{IT}=112,5, \mathrm{IP}=88,9)$. No obstante, la abundancia de roedores histricognatos registrada en el Componente 


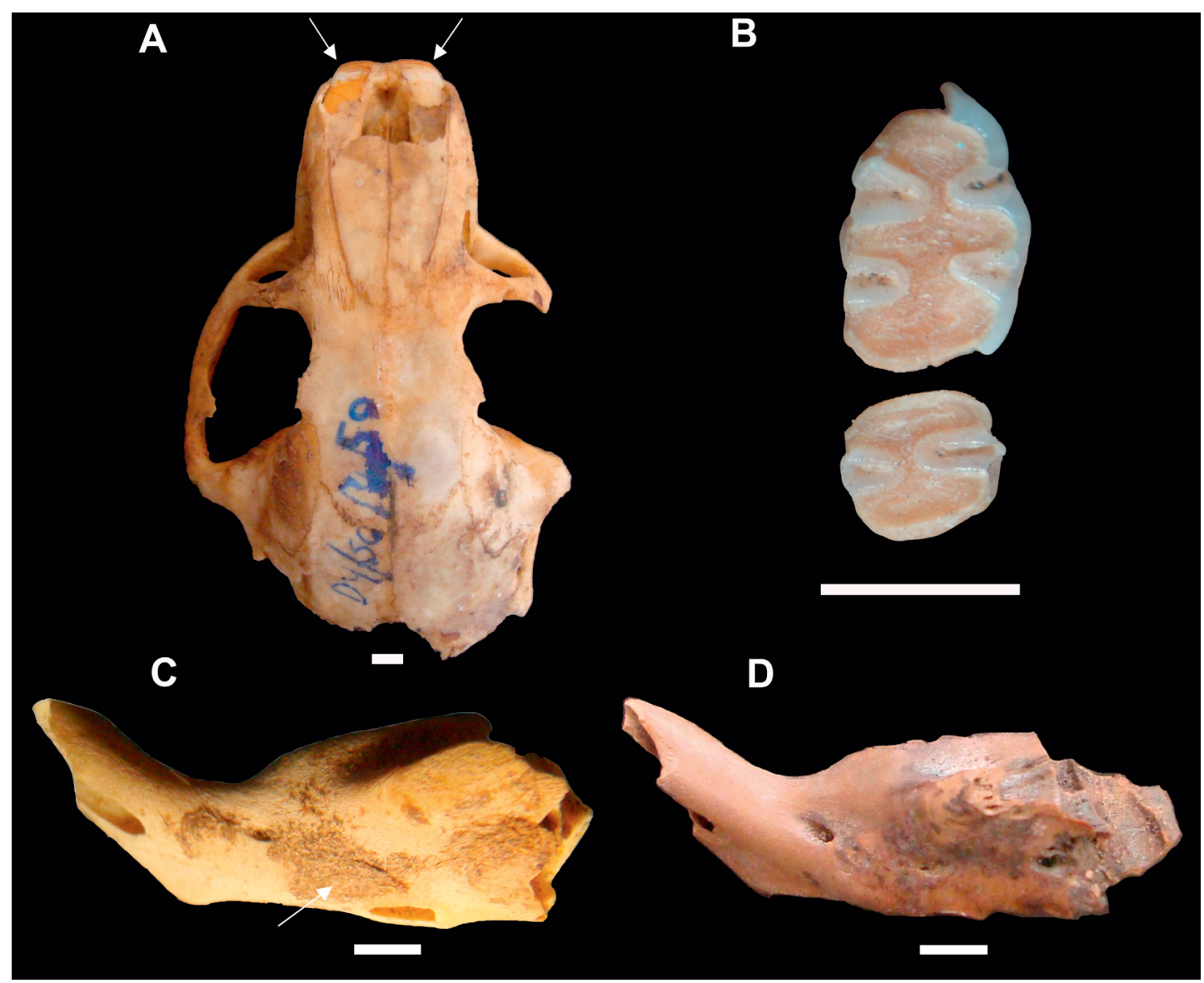

Figura 2. Ejemplos de evidencias tafonómicas en los sitios arqueológicos. (A) Vista dorsal del cráneo de Ctenomys sp., con marcas de corrosión digestiva ligera en los incisivos (CL, Componente III). (B) Vista oclusal de molares superiores (M1 y M2) de Phyllotis xanthopygus, exhibiendo corrosión digestiva moderada (APC). (C) Vista labial de mandíbula de Microcavia australis, con restos de pelos de egagrópilas (CL, Componente V). (D) Vista labial de mandíbula de M. australis, con evidencias de termoalteración (CL, Componente V). Escalas: $2 \mathrm{~mm}$.

Examples of taphonomic processes at the archaeological sites. A. Skull of Ctenomys sp. in dorsal view, with light digestive corrosion in incisors (CL, Component III). (B) Upper molars (M1, M2) of Phyllotis xanthopygus in occlusal view, showing moderate corrosion $(A P C)$. (C) Mandible of Microcavia australis in labial view, with pellet remains (CL, Component V). (D) Mandible of M. australis in labial view, with traces of thermoalteration (CL, Component V). Scales: $2 \mathrm{~mm}$.

I, II y III de CL podría asociarse a sesgos producto de las técnicas de recuperación, debido a que solo se recolectaron los elementos craneales (véase sección Muestras arqueológicas). En relación con lo expuesto, Pardiñas (1999a, 1999b) y Pardiñas y Teta (2013) plantean que los roedores histricognatos presentan cráneos y mandíbulas más robustas que los roedores sigmodontinos y por tanto son más resistentes a procesos postdepositacionales como el pisoteo. Adicionalmente, estudios realizados en Patagonia demostraron que las poblaciones de Ctenomys eran mucho más abundantes en el registro fósil del Pleistoceno/Holoceno, incluso hasta comienzos del siglo XX, respecto de la actualidad. Estas poblaciones se encuentran en franco retroceso desde la introducción del ganado ovino (Ovis aries), el que produce una compactación del suelo por pisoteo y la eventual destrucción de las galerías subterráneas de este roedor fosorial (Pardiñas 1999a; Pardiñas y Teta 2013; Teta et al. 2014). Esta situación pudo ocurrir en Mendoza con el ganado ovino y caprino (Capra hircus), y de este modo podría explicar complementariamente los altos valores de este roedor en los sitios arqueológicos mencionados respecto de los conjuntos actuales de aves rapaces, donde en general no superan el $15 \%$. 
No obstante, las evidencias de elementos con pelos de egagrópilas y con marcas de corrosión digestiva, principalmente ligera, coinciden con los conjuntos de micromamíferos generados por aves Strigiformes y se distancian de aquellos que presentan altos niveles de digestión como los conjuntos producidos por aves Accipritiformes y Falconiformes, mamíferos carnívoros (p.ej., Andrews 1990) e incluso por los grupos humanos (Crandall y Stahl 1995; Deward y Jarardino 2007). A pesar de que las aves Strigiformes son conocidas por generar acumulaciones óseas y dentarias con un alto grado de integridad (Andrews 1990), estudios actuales han demostrado variaciones entre las especies que comprenden este orden. En el área de estudio se encuentran tres especies de Strigiformes con información tafonómica publicada: Tyto alba, Bubo virginianus y Athene cunicularia (Andrews 1990; Fernández et al. 2009; Gómez 2005, 2007; Montalvo y Tejerina 2010). Las tres especies mencionadas, aunque también presentan variaciones en sus dietas, se alimentan principalmente de micromamíferos y ocasionalmente de otros vertebrados e insectos (Bó et al. 2007). Los datos tafonómicos provenientes de los agregados formados por T. alba, concluyen que esta lechuza produce niveles de digestión correspondientes a la categoría ligera (p.ej., Andrews 1990; Fernández et al. 2009; Gómez 2007). Esta lechuza se considera el principal acumulador de restos de micromamíferos en cuevas y refugios rocosos (p.ej., Andrews 1990; Saavedra y Simonetti 1998; Pardiñas 1999a, 1999b; Fernández 2012a). No obstante, Gómez (2005) y Montalvo y Tejerina (2010) registraron que $B$. virginianus y A. cunicularia, respectivamente, causaban mayores niveles de corrosión digestiva en restos de micromamíferos.

Se hallaron algunas evidencias de procesos postdepositacionales, principalmente en el sitio CL. Se registraron tres restos con evidencias de meteorización (categorías I, II y III sensu Andrews 1990), recuperados del Componente I, II y II de CL. En tal sentido, las escasas evidencias de meteorización, la ausencia de bioturbación postdepositacional (p.ej., marcas de raíces y marcas de roedores) y de transporte hídrico, indican que la mayor parte de los restos de micromamíferos exhumados de CL y APC tuvieron un enterramiento rápido y alto grado de conservación (Andrews 1990; Fernández-Jalvo y Andrews 2003; Korth 1979). Sin embargo, los elevados niveles de fractura registrados en los restos óseos de ambos sitios, con ángulos agudos y bordes ásperos, son consistentes con la acción destructiva del pisoteo, frecuentes en cuevas (p.ej., Andrews 1990; Pardiñas 1999a, 1999b). Por otra parte, se recuperaron tres elementos con corrosión sedimentaria en el Componente I, II y III y tres en el Componente IV de CL y se observaron restos con impresiones por óxidos de manganeso en CL (Componente I, II y III= 10,1\%; Componente IV=17,8\%; Componente V= $27,3 \%)$. Ambos procesos se vinculan con posibles eventos de encharcamiento de agua u oscilaciones del nivel freático (Courty et al. 1989).

\section{Comunidades de micromamíferos recientes en el río Grande y áreas circundantes}

Como se exhibe en la Tabla 2, en líneas generales, los conjuntos de micromamíferos de las muestras actuales son dominados por los roedores sigmodontinos Eligmodontia sp. y Phyllotis xanthopygus y el marsupial Thylamys pallidior.

Por un lado, los conjuntos provenientes de los sectores pedemontanos del sudoeste de la provincia de Mendoza muestran una clara vinculación con la estepa patagónica y vegas cordilleranas (Figuras 1 y 3). Estos están representados por los sigmodontinos Euneomys chinchilloides, Abrothrix olivacea, Abrothrix hirta, Chelemys macronyx, Loxodontomys micropus, Reithrodon auritus y Oligoryzomys longicaudatus y el marsupial Lestodelphys halli (Fernández 2012a; Pardinas et al. 2008). Asimismo, se registraron los roedores histricognatos, Ctenomys sp., Microcavia australis y Tympanoctomys barrerae. Por el otro, los conjuntos más alejados del área de estudio, aquellos ubicados en las planicies del este de Mendoza, están caracterizados por especies afines al desierto del Monte (Figuras 1 y 3), destacándose los sigmodontinos Graomys griseoflavus, Calomys musculinus y Akodon dolores y el histricognato Galea leucoblephara (Fernández 2012a; Pardinas et al. 2008).

\section{Comunidades de micromamíferos holocénicas en el río Grande}

En CL, Ctenomys sp. y M. autralis fueron los taxones dominantes en todos los componentes. Además, T. pallidior fue recuperado en todos los componentes, no obstante $L$. halli solo fue encontrado en el Componente IV (Tabla 3).

Analizando la muestra de micromamíferos estudiada por O. Pearson y publicada en Neme 
Tabla 2. Composición taxonómica de las muestras actuales de egagrópilas de aves rapaces. 1. Volcán Malacara. 2. 10 km al Oeste de Bardas Blancas. 3. Cavernas de las Brujas. 4. La Pasarela. 5. Los Frisos. 6. Agua de La Mula. 7. Gruta del Indio. Taxonomic composition of the recent owl pellet samples. 1. Volcán Malacara. 2. $10 \mathrm{~km} \mathrm{~W}$ Bardas Blancas. 3. Cavernas de las Brujas. 4. La Pasarela. 5. Los Frisos. 6. Agua de La Mula. 7. Gruta del Indio.

\begin{tabular}{|c|c|c|c|c|c|c|c|c|c|c|c|c|c|c|}
\hline \multirow{2}{*}{ Taxones / Procedencia } & \multicolumn{2}{|c|}{1} & \multicolumn{2}{|c|}{2} & \multicolumn{2}{|c|}{3} & \multicolumn{2}{|c|}{4} & \multicolumn{2}{|c|}{5} & \multicolumn{2}{|c|}{6} & \multicolumn{2}{|c|}{7} \\
\hline & MNI & $\%$ & MNI & $\%$ & MNI & $\%$ & MNI & $\%$ & MNI & $\%$ & MNI & $\%$ & MNI & $\%$ \\
\hline \multicolumn{15}{|l|}{ Mammalia } \\
\hline \multicolumn{15}{|l|}{ Didelphimorphia } \\
\hline Lestodelphys halli & & & & & 4 & 1,3 & & & & & & & & \\
\hline $\begin{array}{l}\text { Thylamys pallidior } \\
\text { Rodentia }\end{array}$ & 6 & 14,6 & 7 & 7,3 & 17 & 5,5 & 7 & 19,4 & 2 & 12,5 & 1 & 4,5 & 56 & 13,5 \\
\hline Ctenomyidae & & & & & & & & & & & & & & \\
\hline $\begin{array}{l}\text { Ctenomys sp. } \\
\text { Octodontidae }\end{array}$ & 5 & 12,2 & 14 & 14,6 & 43 & 14 & & & 2 & 12,5 & & & 7 & 1,7 \\
\hline $\begin{array}{l}\text { Tympanoctomys barrerae } \\
\text { Caviidae }\end{array}$ & & & & & & & & & 1 & 6,3 & & & & \\
\hline Microcavia australis & 1 & 2,4 & & & 7 & 2,3 & & & 2 & 12,5 & & & 4 & 1 \\
\hline $\begin{array}{l}\text { Galea leucoblephara } \\
\text { Cricetidae }\end{array}$ & & & & & & & & & & & 8 & 36,4 & & \\
\hline Akodon dolores & & & & & & & & & & & 1 & 4,5 & 17 & 4,1 \\
\hline Abrothrix olivacea & 1 & 2,4 & 3 & 3,1 & 14 & 4,5 & 3 & 8,3 & & & & & & \\
\hline Abrothrix hirta & & & 1 & 1 & & & & & & & & & & \\
\hline Abrothrix sp. & & & & & 7 & 2,3 & & & & & & & & \\
\hline Chelemys macronyx & & & & & 6 & 1,9 & & & & & & & & \\
\hline Phyllotis xanthopygus & 8 & 19,5 & 24 & 25 & 80 & 26 & 12 & 33,3 & 2 & 12,5 & 2 & 9,1 & 22 & 5,3 \\
\hline Loxodontomys micropus & & & & & 2 & 0,6 & & & & & & & & \\
\hline Graomys griseoflavus & & & & & & & & & & & 5 & 22,7 & 73 & 17,6 \\
\hline Calomys musculinus & & & & & & & & & & & 2 & 9,1 & 97 & 23,4 \\
\hline Eligmodontia sp. & 19 & 46,3 & 17 & 17,7 & 76 & 24,7 & 12 & 33,3 & 7 & 43,7 & 3 & 13,6 & 137 & 33,1 \\
\hline Oligoryzomys longicaudatus & & & & & & & 1 & 2,8 & & & & & & \\
\hline Oligoryzomys flavescens & & & & & & & & & & & & & 1 & 0,2 \\
\hline Reithrodon auritus & & & & & 1 & 0,3 & & & & & & & & \\
\hline Euneomys chinchilloides & 1 & 2,4 & 30 & 31,3 & 40 & 13 & 1 & 2,8 & & & & & & \\
\hline Euneomys sp. & & & & & 11 & 3,6 & & & & & & & & \\
\hline Total & 41 & & 96 & & 304 & & 36 & & 16 & & 22 & & 414 & \\
\hline
\end{tabular}

et al. (1995), se puede observar un conjunto arqueofaunístico compuesto por marsupiales, roedores histricognatos y sigmodontinos (Tabla 3). Ambas muestras juntas están dominadas por Ctenomys sp., P. xanthopygus, Eligmodontia sp., E. chinchilloides y $T$. pallidior, con la inclusión de $M$. australis y R. auritus. Asimismo, Ctenomys sp., G. leucoblephara y Akodon iniscatus se registraron en todos los componentes arqueológicos y C. musculinus y L. halli en el Componente IV (Tabla 3). Por su parte, en APC se registraron T. pallidior, Ctenomys sp., M. australis, R. auritus, P. xanthopygus y Eligmodontia sp. (Tabla 4).

Las muestras arqueológicas están caracterizadas por bajos valores de MNI (principalmente el Componente V de CL y APC), lo que probablemente sesgue la riqueza y diversidad específica de las mismas, limitando el alcance de las conclusiones.
La dominancia de P. xanthopygus, Eligmodontia sp., E. chinchilloides, T. pallidior, Ctenomys sp. у $M$. australis, tanto en los conjuntos arqueológicos como en localidades actuales próximas a la cuenca media e inferior del río Grande indican que un ambiente con características afines a estepas arbustivas abiertas con alta proporción de suelo desnudo, pedregoso y abundante roca expuesta se mantuvo constante desde comienzos del Holoceno tardío hasta la actualidad. No obstante, los registros de G. leucoblephara, A. iniscatus y C. musculinus únicamente en CL, sugieren condiciones afines al desierto del Monte desde ca. 3.800 a.p. hasta tiempos históricos. Este escenario de mayor aridez es coherente con la ausencia de elementos característicos de vegas cordilleranas en ambas secuencias (A. olivacea, A. hirta, C. macronyx y L. micropus). No obstante, los registros de $R$. auritus en las muestras arqueológicas 

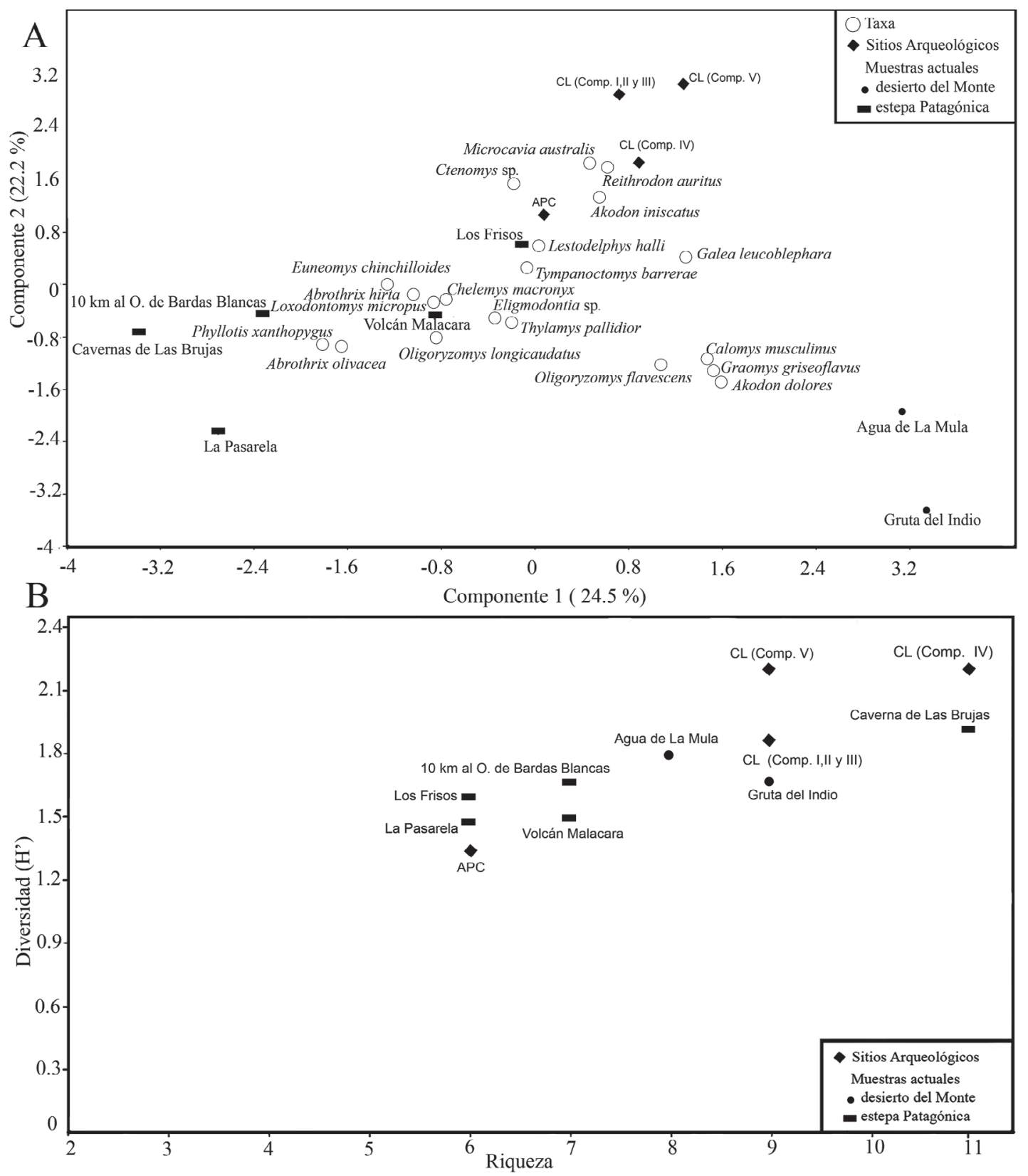

Figura 3. (A) Gráfico de las muestras actuales y arqueológicas (ACP, CL) sobre el plano definido por los ejes 1 y 2 de un Análisis de Componente Principal. (B) Relaciones entre valores de diversidad (H') y riqueza taxonómica de las muestras actuales y arqueológicas. (A) Representation of recent and archaeological (ACP, CL) micromammal samples on the plane defined by axes 1 and 2 of a principal component analysis. $(B)$ Relationship between diversity $\left(H^{\prime}\right)$ and taxonomic richness values of the recent and archaeological samples.

y de L. halli en el Componente IV de CL, señalan un ambiente de mosaico con la estepa patagónica hacia ca. 1.500 a.p.

Teniendo en cuenta los sesgos potenciales por los bajos valores de MNI, los resultados del análisis de componentes principales (Figura 3a) exhiben un ordenamiento de los conjuntos arqueológicos en una posición intermedia, entre las muestras actuales asociadas al desierto del Monte a la derecha y las muestras asociadas a la estepa patagónica hacia la izquierda. En tal sentido, este análisis también nos está indicando una agrupación por filiación 
Tabla 3. Conjuntos de micromamíferos de Cueva de Luna determinados taxonómicamente en este trabajo y por Oliver Pearson (Modificado de Neme et al. 1995:Figura 2).

Small mammal assemblages from Cueva de Luna, taxonomically determined in this work and by Oliver Pearson (Modified from Neme et al. 1995:Figure 2).

\begin{tabular}{|c|c|c|c|c|c|c|c|c|c|c|c|c|c|c|c|c|c|c|}
\hline \multirow{3}{*}{$\begin{array}{c}\text { Procedencia } \\
\text { Taxones }\end{array}$} & \multicolumn{6}{|c|}{ Comp I, II y III } & \multicolumn{6}{|c|}{ Comp IV } & \multicolumn{6}{|c|}{ Comp V } \\
\hline & \multicolumn{2}{|c|}{ Pearson } & \multicolumn{2}{|c|}{$\begin{array}{l}\text { Este } \\
\text { trabajo }\end{array}$} & \multicolumn{2}{|c|}{ Total } & \multicolumn{2}{|c|}{ Pearson } & \multicolumn{2}{|c|}{$\begin{array}{l}\text { Este } \\
\text { trabajo }\end{array}$} & \multicolumn{2}{|c|}{ Total } & \multicolumn{2}{|c|}{ Pearson } & \multicolumn{2}{|c|}{$\begin{array}{l}\text { Este } \\
\text { trabajo }\end{array}$} & \multicolumn{2}{|c|}{ Total } \\
\hline & Nisp & Mni & Nisp & Mni & Mni & $\%$ & Nisp & Mni & Nisp & Mni & Mni & $\%$ & Nisp & Mni & Nisp & Mni & Mni & $\%$ \\
\hline $\begin{array}{l}\text { Mammalia } \\
\text { Didelphimorphia }\end{array}$ & & & & & & & & & & & & & & & & & & \\
\hline $\begin{array}{l}\text { Thylamys pallidior } \\
\text { Lestodelphys halli } \\
\text { Rodentia }\end{array}$ & 6 & 3 & 3 & 2 & 5 & 7,6 & 6 & 3 & $\begin{array}{l}2 \\
3\end{array}$ & $\begin{array}{l}2 \\
2\end{array}$ & $\begin{array}{l}5 \\
2\end{array}$ & $\begin{array}{c}11,4 \\
4,5\end{array}$ & 1 & 1 & 1 & 1 & 2 & 11,8 \\
\hline Ctenomys sp. & 2 & 1 & 48 & 22 & 23 & 34,8 & & & 17 & 8 & 8 & 18,2 & & & 6 & 2 & 2 & 11,8 \\
\hline Caviidae indet. & & & 3 & 3 & 3 & 4,5 & & & 4 & 3 & 3 & 6,8 & & & & & & \\
\hline Microcavia australis & 3 & 2 & 14 & 11 & 13 & 19,7 & & & 2 & 2 & 2 & 4,5 & & & 4 & 3 & 3 & 17,7 \\
\hline $\begin{array}{l}\text { Galea leucoblephara } \\
\text { Cricetidae }\end{array}$ & 16 & 8 & & & 8 & 12,1 & 7 & 4 & & & 4 & 9,1 & 5 & 3 & & & 3 & 17,7 \\
\hline Akodon iniscatus & 2 & 1 & & & 1 & 1,5 & 2 & 1 & & & 1 & 2,3 & 4 & 2 & & & 2 & 11,8 \\
\hline Calomys musculinus & & & & & & & 3 & 2 & & & 2 & 4,5 & & & & & & \\
\hline Phyllotis xanthopygus & 8 & 4 & & & 4 & 6,1 & 1 & 1 & & & 1 & 2,3 & 2 & 1 & & & 1 & 5,9 \\
\hline Eligmodontia sp. & 10 & 5 & & & 5 & 7,6 & 25 & 13 & & & 13 & 29,5 & 4 & 2 & & & 2 & 11,8 \\
\hline Reithrodon auritus & 4 & 2 & & & 2 & 3 & 4 & 2 & & & 2 & 4,5 & 2 & 1 & & & 1 & 5,9 \\
\hline Euneomys chinchilloides & 3 & 2 & & & 2 & 3 & 2 & 1 & & & 1 & 2,3 & 1 & 1 & & & 1 & 5,9 \\
\hline Total & 54 & 28 & 68 & 38 & 66 & & 50 & 27 & 28 & 17 & 44 & & 19 & 11 & 11 & 6 & 17 & \\
\hline
\end{tabular}

ambiental y no por el tipo de ave rapaz generador de las muestras, lo que soporta su condición de buenos estimadores de comunidades de micromamíferos en este estudio. Este patrón taxonómico, con afinidad ambiental de ecotono entre ambos ambientes, se observa claramente en el primer componente principal ( $24,5 \%$ de la varianza) y coincide principalmente con el carácter heterogéneo del Componente IV de $\mathrm{CL}$, acusando los valores de diversidad y riqueza más altos de las muestras arqueológicas y actuales (Figura 3b). En tanto, en el segundo componente (22,2\% de la varianza) esta tendencia no es tan clara, aunque sigue mostrando una diferencia marcada entre las muestras arqueológicas y las muestras actuales.

\section{Paleoambientes, ocupaciones y subsistencia humana en el río Grande}

Desde hace algo más de una década se vienen realizando numerosas investigaciones en el sur de Mendoza y en el extremo norte de Neuquén acerca del registro de los micromamíferos recuperados de contextos arqueológicos, principalmente desde las perspectivas tafonómica y paleoambiental (Fernández 2010, 2012a, 2012b; Fernández et al. 2009, 2012,
Tabla 4. Conjunto de micromamíferos de Alero Puesto Carrasco (APC). Small mammals assemblage from Alero Puesto Carrasco (APC).

\begin{tabular}{lcccc}
\hline \multirow{2}{*}{ Taxones / Procedencia } & \multicolumn{4}{c}{ APC } \\
\cline { 2 - 5 } & NISP & $\%$ & MNI & $\%$ \\
\hline $\begin{array}{l}\text { Mammalia } \\
\text { Didelphimorphia }\end{array}$ & & & & \\
$\begin{array}{l}\text { Thylamys pallidior } \\
\text { Rodentia }\end{array}$ & 3 & 5,3 & 1 & 4,3 \\
Ctenomyidae & & & & \\
Ctenomys sp. & 7 & 12,3 & 3 & 13 \\
$\begin{array}{l}\text { Caviidae } \\
\text { Microcavia australis }\end{array}$ & 4 & 7 & 2 & 8,7 \\
Cricetidae & & & & \\
$\begin{array}{l}\text { Phyllotis xanthopygus } \\
\text { Eligmodontia } \text { sp. }\end{array}$ & 9 & 15,8 & 3 & 13 \\
Reithrodon auritus & 33 & 57,9 & 13 & 56,5 \\
Total & 1 & 1,7 & 1 & 4,3 \\
\hline & 57 & & 23 & \\
\hline
\end{tabular}

2014; Fernández y De Santis 2013; Gasco et al. 2006; Neme et al. 2002). La primera de ellas se centró en determinar los agentes causales de la depositación de los micromamíferos y en el rol que cumplieron en la subsistencia de las poblaciones humanas. La segunda se cimentó en evaluar los 
cambios en las estructuras taxonómicas de los conjuntos de micromamíferos a escalas espacial y temporal y su comparación con las comunidades de micromamíferos que actualmente habitan en la región. De esta forma, se trazaron modelos paleoambientales en los distintos enclaves arqueológicos del sur de Mendoza y extremo norte de Neuquén. Este trabajo continúa con esta línea de investigación regional $y$, en consecuencia, es necesaria su comparación con el registro de micromamíferos de otros sitios arqueológicos ubicados en áreas cercanas.

En la Tabla 5 se expone en forma sintética la principal información paleoambiental obtenida mediante diferentes proxies (polínicos, glaciológicos, sedimentológicos, dendrológicos, malacológicos y micromamíferos) en el sur de Mendoza, norte de Neuquén y Chile Central, desde ca. 8.000 a.p. hasta la actualidad.

A nivel regional diversos autores coinciden que en el sur de Mendoza se establecieron las condiciones climáticas actuales a partir del Holoceno tardío, luego de atravesar durante el Holoceno medio un marcado proceso de aridez en las planicies orientales y un aumento de las precipitaciones níveas en el sector cordillerano (Fernández 2012a; Markgraf 1983; Gil et al. 2005; Navarro et al. 2012; Sting y Garleff 1985; Zárate 2002, Zárate et al. 2010; y literatura citada en estos trabajos). Sin embargo, la estabilidad del Holoceno tardío estuvo interrumpida por fluctuaciones ambientales influenciadas por los eventos El Niño Southern Oscillation (ENSO), procesos de escala global como la Pequeña Edad de Hielo (PEH) entre los siglos XVI-XIX y de deterioro antrópico durante las últimas centurias (p.ej., Fernández 2010, 2012a; Navarro et al. 2012; Villalba 1994; Zárate 2002; Zárate et al. 2010 y literatura allí citada). Esta tendencia paleoambiental también se ha observado en el norte de Neuquén (p.ej., Fernández et al. 2012; Markgraf et al. 2009; Pompei et al. 2012) y en Chile Central (p.ej., de Jang et al. 2013; Jenny et al. 2002; Le Quesne et al. 2009; Villalba 1994).

Como se mencionó previamente para el área del río Grande, hasta este trabajo únicamente se contaba con datos paleoambientales específicos provenientes de la cuenca superior. En este sentido, de acuerdo con el modelo glacial de Mercer (1982), los trabajos de Espizua (2003, 2005) sobre drift (i.e., materiales transportados por los glaciares) identificados a lo largo de los valles El Azufre y El Peñón del sistema río Valenzuela, en la cuenca superior del río Grande, mostraron reavances neoglaciarios durante el Holoceno. El primero de ellos ocurrió entre 5.700 y 4.400 a.p., el segundo avance sucedió entre 2.500 y 2.200 a.p. y el tercero hacia ca. 400 a.p. vinculado con la PEH (Espizua 2003, 2005).

Por su parte, hacia ca. del 1.500 a.p. los conjuntos arqueofaunísticos de la cuenca media del río Grande presentaron especies patagónicas (p.ej., L. halli, R. auritus y E. chinchilloides) y otras afines al desierto del Monte (p.ej., G. leucoblephara, A. iniscatus y C. musculinus), en contraste con las muestras actuales donde principalmente se encontraron taxones patagónicos, señalando un paleoambiente un poco más árido y cálido que el actual. Una hipótesis no necesariamente excluyente para explicar este contexto de transición entre ambos ambientes hacia el Holoceno tardío podría implicar que las especies afines al Monte habrían alcanzado el cauce medio del río Grande, usando el río como corredor biológico. Esta complejidad ambiental en el río Grande ha sido confirmada desde una perspectiva fitogeográfica (Roig et al. 2000), sin embargo, no es del todo clara desde la información emanada de las muestras actuales de micromamíferos documentadas a lo largo del río Grande (Tabla 2; La Pasarela y Los Frisos), donde si bien se observa algún elemento típico del Monte mendocino como $T$. barrerae, la asociación de estas muestras con la unidad patagónica es más acusada. En tanto, en Patagonia central se ha registrado este tipo de comportamiento ecológico en especies de micromamíferos asociadas al Monte (p.ej., T. pallidior, G. griseoflavus, C. musculinus, A. iniscatus), tanto en contextos fósiles como actuales, penetrando en la unidad patagónica mediante los principales sistemas fluviales como el río Chubut (Pardiñas et al. 2011 y literatura allí citada).

En el sector pedemontano del cauce medio del río Grande las primeras ocupaciones humanas se registraron en el sitio Gruta de El Manzano, hacia comienzos del Holoceno medio entre 8.000 y 7.000 a.p. (Gambier 1980, 1985; Neme et al. 2011). Gambier (1980, 1985), describe a esta primera etapa de ocupación como cazadores-recolectores asociados a una industria lítica de tipo Morrillos. Si bien se recuperaron restos de megafauna extinta en la base de la cueva, su asociación con actividades antrópicas ha sido descartada (Neme et al. 2011). Durán (2004), relaciona a esta cultura con una fase biogeográfica de exploración de la región. Sin 
Tabla 5. Síntesis de los diferentes proxies paleoambientales en el sur de Mendoza y Chile Central, desde el 8.000 a.p. hasta la actualidad. Información emanada de este trabajo y recopilada de diversas fuentes (véase el texto). $\mathrm{Ka}=$ mil años; $\mathrm{CH}-1=\mathrm{Cueva}$ Huenul-1; AMA-3= Arroyo Malo-3; LS-3= Laguna El Sosneado-3; CAC=Cueva Arroyo Colorado; $\mathrm{CL}=\mathrm{Cueva}$ de Luna; $\mathrm{APC}=$ Alero Puesto Carrasco; ALM= Agua de La Mula; RA-1= Rincón del Atuel-1; ACA-1= Agua de Los Caballos-1; LP-1= La Peligrosa-1.

Summary of different paleoenvironmental proxies from southern Mendoza and Central Chile, from 8,000 yr. BP to present. The information came from this work and from several other sources (see the text). Ka=thousand year; $\mathrm{CH}-1=\mathrm{Cueva}$ Huenul-

1; $A M A-3=$ Arroyo Malo-3; $L S$-3= Laguna El Sosneado-3; $C A C=$ Cueva Arroyo Colorado; $C L=$ Cueva de Luna; $A P C=$

Alero Puesto Carrasco; $A L M=$ Agua de La Mula; $R A-1=$ Rincón del Atuel-1; $A C A-1=$ Agua de Los Caballos-1; $L P-1=$ La Peligrosa-1.

\begin{tabular}{lll}
\hline & $8.000-4.000$ años a.p. & 4.000 años a.p. hasta la actualidad \\
\hline \multirow{2}{*}{ Chile Central } & $\begin{array}{l}\text { ca. } 5 \text { ka clima más árido y cálido } \\
\text { que el actual (Lag. Aculeo) }\end{array}$ & $\begin{array}{l}\text { Condiciones más húmedas, semejantes al presente. } \\
\text { Variaciones asociadas a ENSO (Lag. Aculeo) }\end{array}$
\end{tabular}

Multiproxy

Oscilaciones frías-áridas/cálidas-húmedas asociadas a eventos

Multiproxy ENSO y PEH (Lag. Chepical, Glaciar Cipreses)

Norte de Neuquén

Micromamíferos

Multi-proxy

Sur de Mendoza

(Altoandina)

Polínico

Glaciológico

(Patagónica)
5-3,8 ka menor heterogeneidad ambiental (Caverna de las Brujas). Estepas arbustivas abiertas, rocoso y pedregoso (AMA-3)
1,4 ka mosaico de estepas arbustivas, peladales y áreas rocosas abiertas (CH-1)

ca. 2-1 ka aumento de humedad y actividad del fuego (Mallín Vaca Lauquén). 1.5 ka condiciones de ecotono Monte-Patagonia (CH-1)

Condiciones similares a las actuales, gran variabilidad ambiental influenciada por ENSO. 2,8 ka mayor disponibilidad hídrica (Agua Buena)

5,7-4,4 ka avances glaciarios (alto valle río Grande)

2,5-2,2 ka y 0,4 ka reavances glaciarios (alto valle río Grande)

3,8-2,2 ka aumento de la arbustización. 2,2-0,1 ka continúa el ambiente abierto, pedregoso y rocoso (AMA-3). 2,1-0,6 ka ambiente abierto, pedregoso,

rocoso y con vegas. 0,6 ka un retroceso de vegas (LS-3).

4 ka ambiente estepas arbustivas abiertas, rocoso y pedregoso (Cueva Palulo). 3,2-0 ka ambiente abierto, pedregoso y rocoso. 1,4-0,77 ka mayor disponibilidad hídrica (CAC). 3,8 ka ambiente abierto, rocoso y pedregoso, relacionado al Monte. 3,8 ka ambiente abierto, pedregoso y rocoso, asociado al Monte (CL, APC). 1,5 ka ecotono Monte-Patagonia (CL). Actualmente, ambiente con deterioro antrópico

Polínico

3 ka aumento de la temperatura. Condiciones similares a las actuales (turbera en río Salado)

6-4,5 ka avances glaciarios (alto valle Río Atuel). 5,7-4,4 ka avances glaciarios (alto valle río Grande)

3 ka retracción glaciar (alto valle río Atuel). 2,5-2,2 ka y 0,4 ka reavances glaciarios (alto valle río Grande)

6,4-1,9 ka idem Holoceno medio. 1,9-0,5 ka aridez y mayor temperatura, vegetación ecotono Monte-

6,4-1,9 ka condiciones más Sedimentológico húmedas y frías que el presente (Lag. El Sosneado)
Patagonia. 0,5 ka más humedad, vegetación ecotono Monte-Patagonia (Lag. El Sosneado). 4,1-2,8 ka desarrollo de vegas. 2,8 ka menor disponibilidad hídrica (Agua Buena). 3,8-1,4 ka microambiente húmedo (Vega de la Cueva)
Cuerpo de agua muy somero y vegetado de tipo palustre, ambiente semejante al actual (Puesto Moya, ca. Lag. Llancanelo) 
continuación Tabla 5.

\begin{tabular}{lll}
\hline & $8.000-4.000$ años a.p. & 4.000 años a.p. hasta la actualidad \\
\hline $\begin{array}{ll}\text { Monte) } \\
\text { Micromamíferos }\end{array}$ & $\begin{array}{l}1,6-1 \text { ka ecotono Monte-Patagonia (ALM). 1,5-0,25 } \\
\text { ka taxones asociados al Monte (RA-1, ACA-1, LP-1) }\end{array}$ \\
\cline { 2 - 3 } Polínico & $\begin{array}{l}\text { 5 ka vegetación del Monte } \\
\text { (Gruta del Indio) }\end{array}$ & $\begin{array}{l}\text { Vegetación del Monte, como en la actualidad. 1,6-1,2 ka ecotono } \\
\text { Monte-Patagonia (Gruta del Indio) }\end{array}$ \\
\cline { 2 - 3 } Sedimentológico & $\begin{array}{l}\text { Reactivación eólica asociada a } \\
\text { mayor aridez (planicie sanrafaelina) }\end{array}$ & \\
\cline { 2 - 3 } Malacológico & Condiciones más áridas que las & \\
& actuales (Villa Atuel =La Guevarina) &
\end{tabular}

embargo, Neme (2007) y Neme et al. (2011) sugieren que estaríamos frente a una etapa de colonización del área, debido a la observación de tasas más altas de depositación de materiales, especialmente líticos, óseos y moluscos, respecto de los componentes más tardíos. En coincidencia con otras áreas del sur de Mendoza, existe un hiatus ocupacional en Gruta El Manzano ca. 7.000 y 2.100 a.p., el que fue vinculado a un proceso de aridez del Holoceno medio y erupciones volcánicas (Durán y Mikkan 2009; Neme et al. 2011). Gambier $(1980,1985)$ definió a las ocupaciones correspondientes al Holoceno tardío como la etapa Agroalfarera. Por su parte, Durán $(1997,2004)$ elabora un modelo regional de esta área a base del registro arqueológico de Gruta El Manzano y de otros reparos cercanos al mismo (CL, APC y Cañada de Cachi). Estos últimos presentan secuencias asociadas únicamente para el Holoceno tardío y tienen una marcada ocupación humana con alta densidad de materiales hacia los últimos 2.000 a.p. Los autores mencionados plantean que el valle medio del río Grande pudo funcionar como un límite interétnico, donde se dieron importantes contactos entre los pobladores de la Payunia y los de la cordillera, en un contexto de un modelo de variación estacional. Contrariamente, Borrero (2002) argumenta que en regiones áridas los valles fluviales podrían funcionar como refugio para las distintas poblaciones de áreas circundantes.

Asimismo, para explicar el hiatus ocupacional observado en CL entre ca. 3.800 y 1.500 años a.p. se plantearon hipótesis relacionadas a las erupciones volcánicas y factores ambientales desfavorables (p.ej., Durán 2004). Coincidentemente esto podría asociarse con las condiciones de mayor aridez imperantes a comienzos del Holoceno tardío, evidenciadas desde el registro de micromamíferos de este sitio. Por otra parte, el mejoramiento ambiental registrado hacia ca. 1.500 a.p. marcado por condiciones ecotonales entre Monte y estepa patagónica, podría relacionarse con el período de ocupaciones humanas prehispánicas (Componente IV) más continuas e intensivas observadas en CL (Durán 1997, 2004; Durán y Ferrari 1991; Neme et al. 1995). En forma comparable, entre los 1600 y 1.000 años a.p. los conjuntos de micromamíferos recuperados del sitio arqueológico Agua de la Mula, ubicado en las planicies orientales del sur de Mendoza, indicaron un mejoramiento ambiental, pasando de condiciones de Monte a la de transición Monte y estepa patagónica (Fernández 2010, 2012a).

A base de la información arqueológica Neme et al. (2011) señalan que un proceso de intensificación de los recursos tuvo lugar en esta área hacia el 2.000 a.p., en forma parangonable con el alto valle del río Atuel (Neme 2007). Estos autores observaron una disminución en la explotación de L. guanicoe, un aumento en el consumo de la fauna de mediano tamaño y de los recursos vegetales (véase también Giardina 2010a, 2010b; Llano 2011). No obstante, este proceso no se corroboró tafonómicamente desde los conjuntos de micromamíferos recuperados de CL y APC. Congruentemente, en el sur de Mendoza y extremo norte de Neuquén se ha demostrado que las aves rapaces fueron las principales responsables de las acumulaciones de micromamíferos en sitios arqueológicos (Fernández 2010, 2012a, 2012b; Fernández y De Santis 2013; Fernández et al. 2012, 2014; Gasco et al. 2006; Neme et al. 2002). Para esta región únicamente se han registrado evidencias fehacientes de explotación antrópica de micromamíferos en el sitio arqueológico Cueva Arroyo Colorado hacia ca. 1.400 años a.p., ubicado cerca de las nacientes del río Salado (Fernández 2012a; Fernández et al. 2009). Llamativamente esta situación contrasta con lo registrado en otras 
regiones linderas de Argentina como en las Sierras Centrales, Pampa y Patagonia (p.ej., Acosta y Pafundi 2005; Fernández, del Papa et al. 2011; Medina et al. 2011, 2012; Pardiñas 1999a, 1999b; Quintana 2005; Quintana et al. 2002; Teta et al. 2005) y en Chile Central (p.ej., Saavedra 1994; Simonetti y Cornejo 1991).

\section{Consideraciones Finales}

Este trabajo es parte de una línea de investigación iniciada hace algo más que una década acerca de los micromamíferos recuperados de sitios arqueológicos del sur de Mendoza y extremo norte de Neuquén. Las contribuciones emanadas de esta línea de investigación han tenido un impacto sustancial en la arqueología y paleoecología regional, principalmente respondiendo a interrogantes respecto del rol que cumplieron los micromamíferos en las economías de los grupos de cazadores y recolectores y a cerca de su utilización como indicadores indirectos de condiciones paleoambientales.

Las características tafonómicas de las muestras indicaron que los conjuntos de micromamíferos de CL y APC fueron depositados principalmente por la acción de aves Strigiformes, posiblemente Tyto alba. Por ende, los grupos humanos que habitaron ambos sitios no intervinieron en la génesis de los conjuntos de micromamíferos. Esto contrasta con el patrón esperado de consumo humano de presas de bajo retorno calórico como sugiere el proceso de intensificación de los recursos planteado para la región. Esto no indica necesariamente que el proceso de intensificación de los recursos no haya tenido lugar en el sur de Mendoza debido a que el mismo se ha demostrado en trabajos previos. Este proceso se pudo dar con distinta intensidad, o posiblemente para las sociedades de cazadores-recolectores no fue necesario la explotación de los micromamíferos, los que se encuentran más bajo en el ranking de recursos de la región, ya sea por la falta de una tecnología adecuada para su caza, tabúes, o por la escasez de especies con comportamientos gregarios que faciliten el incremento de su rendimiento mediante prácticas de captura masiva.

Los escasos restos con meteorización, marcas de raíces, marcas de roedores y de transporte hídrico, señalan que los conjuntos tuvieron un enterramiento rápido y alto grado de conservación. Sin embargo, se encontraron evidencias de pisoteo, corrosión sedimentaria y óxido de manganeso, sugiriendo posibles eventos de encharcamiento de agua u oscilaciones del nivel freático de moderado impacto.

$\mathrm{El}$ análisis de los conjuntos de micromamíferos recuperados de CL y APC señala un ambiente con características afines a estepas arbustivas abiertas con alta proporción de suelo desnudo, pedregoso y abundante roca expuesta, que se mantuvo constante desde ca. 3.800 a.p. hasta la actualidad. Las características de ambiente de Monte se mantienen durante toda la secuencia en CL. Sin embargo, hacia ca. 1.500 a.p. hay evidencias de mejoramiento ambiental con taxones afines al Monte y patagónica, coincidente con el período de ocupaciones humanas prehispánicas más continuas e intensivas en CL.

Finalmente se destaca la necesidad de aumentar la cantidad de muestras arqueológicas y actuales de micromamíferos en el cauce medio del río Grande, para poder discutir con más detalle las ideas planteadas en esta contribución. En la misma línea, resulta necesario incrementar la utilización de otros indicadores paleoambientales indirectos en el área (p.ej., polínicos, sedimentológicos, malacológicos).

Agradecimientos: A Víctor Durán por permitirnos estudiar las muestras arqueológicas y a Clelia Mosto por ceder la muestra de egagrópilas de Volcán Malacara. A la UNLP (proyecto 11/N601) y al CONICET por el soporte institucional y económico. Cuatro revisores anónimos aportaron valiosos comentarios y sugerencias que ayudaron a mejorar la calidad y claridad de este trabajo.

\section{Referencias Citadas}

Acosta, A. y L. Pafundi 2005. Zooarqueología y tafonomía de Cavia aperea en el humedal del Paraná inferior. Intersecciones en Antropología 6:59-74.

Andrews, P. 1990. Owls, Caves and Fossils. University of Chicago Press, Chicago.

Andrews, P. 1995. Mammals as palaeoecological indicators. Acta Zoológica Cracovensia 38:59-72.
Bettinger, R. 1991. Aboriginal occupation at high altitude: Alpine villages in the White Mountains of Eastern California. American Anthropologist 93:657-679.

Bó, M.S., A.V. Baladrón y L.M. Biondi 2007. Ecología trófica de Falconiformes y Strigiformes: tiempo de síntesis. Hornero 22:97-115.

Borrero, L.A. 2002. Arqueología y biogeografía humana en el sur de Mendoza (comentario crítico). En Entre Montañas y 
Desiertos: Arqueología del Sur de Mendoza, editado por A. Gil y G. Neme, pp. 195-102. Sociedad Argentina de Antropología, Buenos Aires.

Broughton, J.M. 1994. Late Holocene resource intensification in the Sacramento Valley: The vertebrate evidence. Journal of Archaeological Science 21:501-514.

Cabrera, A.L. 1976. Regiones fitogeográficas argentinas. Enciclopedia Argentina de Agricultura y Jardinería 1:1-85.

Capitanelli, R.G. 1972. Geomorfología y Clima de la provincia de Mendoza. En Geología, Geomorfología, Climatología, Fitogeografía y Zoogeografía de la provincia de Mendoza, editado por F.A. Roig, pp. 15-48. Reedición especial del Boletín de la Sociedad Argentina de Botánica 8, Mendoza.

Courty, M.A., P. Goldberg y R. Macphail 1989. Soil and Micromorphology in Archaeology. Cambridge University Press, Cambridge.

Crandall, B.D y P.W. Stahl 1995. Human digestive effects on a micromammalian skeleton. Journal of Archeological Science 22:789-797.

de Jong, R., L. von Gunten, A. Maldonado y M. Grosjean 2013. Late Holocene summer temperatures in the central Andes reconstructed from the sediments of high-elevation Laguna Chepical, Chile ( $\left.32^{\circ} \mathrm{S}\right)$. Climate Past 9:1921-1932.

Deward, G. y A. Jarardino 2007. Micromammals: when humans are the hunters. Journal of Taphonomy 5:1-14.

Durán, V. 1997. Arqueología del Valle del Río Grande, Malargüe, Mendoza. Tesis Doctoral. Facultad de Ciencias Naturales y Museo, Universidad Nacional de La Plata, La Plata.

Durán, V. 2004. Poblaciones Indígenas de Malargüe: su Arqueología e Historia. CEIDER, Universidad Nacional de Cuyo, Segunda reimpresión, Mendoza.

Durán, V. y J. Ferrari 1991. El proceso de araucanización en el sur mendocino desde una perspectiva arqueológica. Actas del XI Congreso Nacional de Arqueología Chilena Vol. 3, pp. 165-188. Museo de Historia Natural, Santiago.

Durán, V., G. Neme y A. Gil 1999. Algunos problemas relacionados con el registro arqueológico de alero Puesto Carrasco (curso medio del valle del río Grande, Malargüe, Mendoza). En Soplando en el Viento, Actas de las Terceras Jornadas de Arqueología de la Patagonia, editado por J.B. Belardi, pp. 333-356. Bariloche.

Durán, V. y R. Mikkan 2009. Impacto del volcanismo holocénico sobre el poblamiento humano del sur de Mendoza (Argentina). Intersecciones en Antropología 10:295-310.

Espizua, L. 2003. Holocene glaciar fluctuations in the south of Mendoza Andes, Argentina. Actas II Congreso Argentino de Cuaternario y Geomorfología, pp. 87-92. Universidad Nacional de Tucumán, Tucumán.

Espizua, L. 2005. Holocene glacier chronology of Valenzuela Valley, Mendoza Andes, Argentina. The Holocene 15:1079-1085.

Fernández, F.J. 2010. Paleozoogeography of the wine mouse (Akodon oenos) \& late Holocene paleoenvironments in southcentral of Mendoza, Argentina. Ethnobiology Letters 1:52-57.

Fernández, F.J. 2012a. Microvertebrados del Holoceno de Sitios Arqueológicos en el Sur de Mendoza (República Argentina):
Aspectos Tafonómicos y sus Implicancias en la Subsistencia Humana. Tesis de Doctoral. Facultad de Ciencias Naturales y Museo, Universidad Nacional de La Plata, Editorial SeDiCI, La Plata.

Fernández, F.J. 2012b. Tafonomía de microvertebrados exhumados del sitio arqueológico Agua de los Caballos-1 (Mendoza, Argentina). Comechingonia 16:169-174.

Fernández, F.J., F. Ballejo, G. Moreira, E. Tonni y L. De Santis 2011. Roedores Cricétidos de la Provincia de Mendoza. Guía Craneodentaria Orientada para su Aplicación en Estudios Zooarqueológicos. Sociedad Argentina de Antropología y Universitas Sarmiento, Córdoba.

Fernández, F.J., L. Del Papa, G.J. Moreira, L. Prates y L.J.M. De Santis 2011. Small mammal remains recovered from two archaeological sites in the middle and lower Negro River valley (Late Holocene, Argentina): Taphonomic issues and paleoenvironmental implications. Quaternary International 245:136-147.

Fernández, F.J. y L.J.M. De Santis 2013. Tafonomía de restos de micromamíferos recuperados del sitio arqueológico Agua de La Mula (Mendoza, Argentina) y su implicancia en la subsistencia humana. Cuadernos del INAPAL, Series Especiales 1:181-195.

Fernández, F.J., L.J.M. De Santis, G. Neme 2014. Micromammals, taphonomy, paleoenvironments and human occupation during the Holocene in Arroyo Malo-3 (west-central Argentina). Archaeofauna (en prensa).

Fernández, F.J., G.J. Moreira, G.A. Neme y L.J.M. De Santis 2009. Microvertebrados exhumados del sitio arqueológico "Cueva Arroyo Colorado" (Mendoza, Argentina): aspectos tafonómicos y significación paleoambiental. Archaeofauna 18:99-118.

Fernández, F.J., P. Teta, R. Barberena y U.F.J. Pardiñas 2012. Small mammal remains from Cueva Huenul 1, Argentina. Taphonomy and paleoenvironments since the Late Pleistocene. Quaternary International 278:22-31.

Fernández-Jalvo, Y. y P. Andrews 1992. Small mammal taphonomy of Gran Dolina, Atapuerca (Burgos), Spain. Journal of Archaeological Science 19:407-428.

Fernández-Jalvo, Y. y P. Andrews 2003. Experimental effects of water abrasion on bone fragments. Journal of Taphonomy $1: 147-163$.

Gambier, M. 1980. Excavaciones arqueológicas en la Gruta de El Manzano, Río Grande, Mendoza. Boletín del Museo de Ciencias Naturales y Antropológicas “Juan Cornelio Moyano” 1:45-55.

Gambier, M. 1985. La Cultura de los Morrillos. Instituto de Investigaciones Arqueológicas y Museo Facultad de filosofía, humanidades y Artes, Universidad Nacional de San Juan, San Juan.

Gasco, A., M.I. Rosi y V. Durán 2006. Análisis arqueofaunístico de microvertebrados en "Caverna de las Brujas" (MalargüeMendoza-Argentina). Anales de Arqueología y Etnología, Volumen especial 61:135-162.

Giardina, M. 2010a. Registro zooarqueológico en el Alero Puesto Carrasco: un aporte para discutir la explotación de reidos en Nordpatagonia. En Zooarqueología a Principios del Siglo XXI: Aportes Teóricos, Metodológicos y Casos de Estudio, editado por M. De Nigris, P.M. Fernández, M. Giardina, A. Gil, M.A. 
Gutiérrez, A. Izeta, G. Neme y H.D. Yacobaccio, pp. 133-143. Ediciones del Espinillo, Buenos Aires.

Giardina, M. 2010b. Rheidae human exploitation in North Patagonia, Argentina (South America). Groningen Archaeological Studies 10:97-102.

Gil, A., M. Zárate y G. Neme 2005. Mid-Holocene Paleoenvironments and the Archaeological record of Southern Mendoza, Argentina. Quaternary International 132:81-94.

Gómez, G.N. 2005. Analysis of bone modification of Bubo virginianus pellets from Argentina. Journal of Taphonomy 3:1-16.

Gómez, G.N. 2007. Predators categorization based on taphonomic analysis of micromammals bone: a comparison to proposed models. En Taphonomy and Zooarchaeology in Argentina, editado por M.A Gutiérrez, L. Miotti, G. Barrientos, G. Mendoni Goñalons y M. Salemme, pp. 1-16. BAR International Series 1601, Oxford.

Grayson, D.K. 1984. Quantitative Zooarchaeology: Topics in the Analysis of Archaeological Faunas. Academics Press, Orlando.

Hammer, Ø. 1999-2011. PAST PAleontological STatistics Version 2.07. Reference manual. Natural History Museum, University of Oslo, Oslo.

Jenny, B., B.L. Valero-Garcés, R. Villa-Martínez, R. Urrutia, M. Geyh y H. Veit 2002. Early to Mid-Holocene aridity in Central Chile and the Southern Westerlies: The Laguna Aculeo record (34 S). Quaternary Research 58:160-170.

Korth, W. 1979. Taphonomy of Microvertebrate Fossil Assemblages. Annals of Carnegie Museum 15:235-285.

Le Quesne, C., C. Acuña, J.A. Boninsegna, A. Rivera y J. Barichivich 2009. Long-term glacier variations in the Central Andes of Argentina and Chile, inferred from historical records and tree-ring reconstructed precipitation. Palaeogeography, Palaeoclimatology, Palaeoecology 281:334-344.

Llano, C. 2011. Aprovechamiento de los Recursos Vegetales entre las Sociedades Cazadoras-Recolectoras del Sur de Mendoza. Tesis Doctoral. Centro Regional Universitario Bariloche, Universidad Nacional del Comahue, Bariloche.

Markgraf, V. 1983. Late and postglacial vegetational and paleoclimatic change in subantartic and arid environments in Argentina. Palynology 7:43-70.

Markgraf, V., C. Whitlock, R.S. Anderson y A. García 2009. Late Quaternary vegetation and fire history in the northernmost Nothofagus forest region: Mallín Vaca Lauquen, Neuquén Province, Argentina. Journal of Quaternary Science 24:248-258.

Martínez Carretero, E. 2006. Payunia Reserva Natural y Provincia Fitogeográfica. Arqueología y Ambiente de Áreas Naturales Protegidas de la Provincia de Mendoza. Anales de Arqueología y Etnología 61:217-235.

Medina, M., D. Rivero y P. Teta 2011. Consumo antrópico de pequeños mamíferos en el Holoceno de Argentina central: perspectivas desde el abrigo rocoso Quebrada del Real 1 (Pampa de Achala, Córdoba). Latin American Antiquity 22:618-631.

Medina, M., P. Teta y D. Rivero 2012. Burning damage and small-mammal human consumption in Quebrada del Real 1 (Cordoba, Argentina): an experimental approach. Journal of Archaeological Science 39:737-743.
Mercer, J.H. 1982. Holocene glacier variations in Southern South America. Striae 8:35-40.

Montalvo, C.I. y P. Tejerina 2010. Análisis tafonómico de los huesos de anfibios y roedores depredados por Athene cunicularia (Strigiformes, Strigidae) en La Pampa, Argentina. En Mamül Mapu: Pasado y Presente desde la Arqueología Pampeana, editado por M. Berón, L. Luna, M. Bonomo, C.I. Montalvo, C. Aranda y M. Carrera Aizpitarte, pp. 323-334. Libros del Espinillo, Buenos Aires.

Navarro, D., L.D. Rojo, C.G. De Francesco y G.S. Hassan 2012. Paleoecología y reconstrucciones paleoambientales en Mendoza durante el Holoceno. En Paleocología Humana en el Sur de Mendoza: Perspectivas Arqueológicas, editado por Neme G. y A. Gil, pp. 17-56. Sociedad Argentina de Antropología, Buenos Aires.

Neme, G. 2007. Cazadores Recolectores de Altura en los Andes Meridionales: el Alto Valle del Río Atuel. British Archaeological Reports 1591, Archaeopress, Oxford.

Neme, G., V. Durán y A. Gil 1995. Análisis arqueofaunístico del sitio Cueva de Luna (Malargüe-Mendoza, Argentina). Actas XIII Congreso Nacional de Arqueología Chilena. Hombre y Desierto, pp. 363-370. Universidad de Antofagasta, Antofagasta.

Neme, G., A. Gil y V. Durán 1999. El registro arqueofaunístico del sitio Alero Puesto Carrasco (Malarguie; Mendoza). En Soplando en el Viento, Actas de las terceras Jornadas de Arqueología de la Patagonia, editado por J.B. Belardi, pp. 491-514. Bariloche.

Neme, G., G. Moreira, A. Atencio y L.J.M. De Santis 2002. El registro de microvertebrados del sitio arqueológico Arroyo Malo 3 (provincia de Mendoza, Argentina). Revista Chilena de Historia Natural 75:409-421.

Neme, G., A. Gil, R. Garvey, C. Llano, A. Zangrando, F. Franchetti, C. De Francesco y T. Michieli 2011. El registro arqueológico de la Gruta El Manzano y sus implicancias para la arqueología de Nordpatagonia. Magallania 39:243-265.

Pardiñas, U.F.J. 1999a. Los Roedores Muroideos del Pleistoceno Tardío-Holoceno en la Región Pampeana (Sector Este) y Patagonia (República Argentina): Aspectos Taxonómicos, Importancia Bioestratigráficas y Significación Paleoambiental. Tesis Doctoral. Facultad de Ciencias Naturales y Museo, Universidad Nacional La Plata, la Plata.

Pardiñas, U.F.J. 1999b. Tafonomía de microvertebrados en yacimientos arqueológicos de Patagonia. Arqueología 9:265-308.

Pardiñas, U.F.J. y P. Teta 2013. Holocene stability and recent dramatic changes in micromammalian communities of northwestern Patagonia. Quaternary International 305:127-140.

Pardiñas, U.F.J., P. Teta, G. D’Elía y E.P. Lessa 2011. The evolutionary history of sigmodontine rodents in Patagonia and Tierra del Fuego. Biological Journal of the Linnean Society 103:495-513.

Pardiñas, U.F.J., P. Teta y D.E. Udrizar Sauthier 2008. Mammalia, Didelphimorphia and Rodentia Southwest of the province of Mendoza, Argentina. Check List 4:218-225.

Pearson, O.P. 1995. Annotated keys for identifyng small mammals living in or near Nahuel Huapi National Park or Lanin National Park southern Argentina. Mastozoología Neotropical 2:99-148. 
Pompei, M.P., R. Barberena, M.E. De Porras, K. Borrazzo, A. Rughini y A. Gil 2012. Late Quaternary Ecosystems and Humans in Northern Patagonia (Neuquén, Argentina). Southbound. Late Pleistocene Peopling of Latin America. Current Research in the Pleistocene:187-190.

Quintana, C.A. 2005. Despiece de microrroedores en el Holoceno Tardío de las Sierras de Tandilia (Argentina). Archaeofauna 217:227-241.

Quintana, C.A., F. Valverde y D.L. Mazzanti 2002. Roedores y lagartos como emergentes de la diversificación de la subsistencia durante el Holoceno tardío en sierras de la región Pampeana Argentina. Latin American Antiquity 4:455-473.

Roig, V.G. 1972. Esbozo general del poblamiento animal en la provincia de Mendoza. En Geología, Geomorfología, Climatología, Fitogeografía y Zoogeografía de la Provincia de Mendoza, editado por F.A. Roig, pp. 81-88. Reedición especial del Boletín de la Sociedad Argentina de Botánica 8, Mendoza.

Roig, F., E. Martínez Carretero y E. Méndez 2000. Vegetación de la provincia de Mendoza. En Recursos y Problemas Ambientales de la Zona Árida. Provincias de Mendoza, San Juan y La Rioja, Argentina, editado por E.M. Abraham y F.R. Martínez, pp. 63-70. Programa de Cooperación para la Investigación, Junta de Gobierno de Andalucía y Universidades y Centros de Investigación de la Región Andina Argentina, Andalucía.

Saavedra, B. 1994. Tafonomía de micromamíferos de Chile central. Actas del II Taller de Arqueología de Chile Central Tomo 1:1-22.

Saavedra, B. y J.A Simonetti 1998. Small mammals taphonomy: Intraspecific bone assemblage comprasion between South and North American Barn Owl, Tyto alba, populations. Journal of Archaeological Science 25:165-170.
Simonetti, J.A y L.E. Cornejo 1991. Archaeological evidence of rodent consumption in central Chile. Latin American Antiquity 2:92-96.

Sting, H. y K. Garleff 1985. Glacier variations and climate of the late quaternary in the subtropical and mide- latitude andes of Argentina. Aeitschrift für Gletscherkunde 21:225-228.

Teta, P., A. Andrade y U.F.J. Pardiñas 2005. Micromamíferos (Didelphimorphia y Rodentia) y paleoambientes del Holoceno tardío en la Patagonia noroccidental extraandina (Argentina). Archaeofauna 14:183-197.

Teta, P., A. Formoso, M. Tammone, D.C. de Tommaso, F.J. Fernández, J. Torres y U.F.J. Pardiñas 2014. Micromamíferos, cambio climático e impacto antrópico: ¿Cuánto han cambiado las comunidades del sur de América del Sur en los últimos 500 años? Therya 5:7-38.

Villalba, R. 1994. Fluctuaciones climáticas en latitudes medias de América del Sur durante los últimos 1.000 años: sus relaciones con la Oscilación del Sur. Revista Chilena de Historia Natural 67:453-461.

Zárate, M. 2002. Los ambientes del tardiglacial y Holoceno en Mendoza. En Entre Montañas y Desiertos: Arqueología del Sur de Mendoza, editado por A. Gil y G. Neme, pp. 9-42. Sociedad Argentina de Antropología, Buenos Aires.

Zárate, M., A. Gil y G. Neme 2010. Integrando los registros: generalizaciones, alcances y limitaciones. En Condiciones paleoambientales y ocupaciones humanas del centro-oeste de Argentina durante la transición Pleistoceno-Holoceno y Holoceno de Mendoza, editado por M. Zárate, A. Gil y G. Neme, pp. 309-330. Sociedad Argentina de Antropología, Buenos Aires. 
